\title{
Peritoneal dialysis in congestive heart failure
}

Authors: Abirami Krishnan, D.G. Oreopoulos

ABSTRACT Heart failure is a major and growing health problem. There have been major advances in the understanding of pathophysiology of heart failure as a chronic progressive disorder which has led to newer therapies. Whatever the etiology, many heart failure patients eventually progress to a refractory stage characterized by worsening renal function and resistance to diuretic therapy with attending severe edema. A logical treatment for this "cardiorenal syndrome" is the use of dialysis, which is efficient in treating both the hypervolaemia and azotaemia of refractory heart failure. Though all modalities of dialysis have been tried, peritoneal dialysis (PD) is the simplest choice and offers several advantages. It is an already established long-term home-based therapy and does not require complex machinery or hospital resources. PD is associated with preservation of residual renal function, gentle continuous ultrafiltration, hemodynamic stability, better middle molecule clearance, sodium seiving with maintenance of normonatremia and maybe less inflammation, especially with newer solutions, compared to hemodialysis. In this paper we discuss the potential advantages of PD in the treatment of heart failure, review available literature and lay some foundation for future research.

\section{EPIDEMIOLOGY}

Heart failure (HF) is a major and growing public health problem in the United States. Overall incidence of congestive heart failure (CHF) approaches 10// 000 after age of 65 (Framingham Heart Study data) and increases with increasing age. The disorder is the primary reason for 12 to 15 million office visits and 6.5 million hospital days each year. In 2005 alone, the cost of management of heart failure was \$27.9 billion in the US. ${ }^{(1)}$

Though multiple clinical trials completed during the past 15 years have unequivocally shown a substantial reduction in mortality for patients with systolic heart failure, large epidemiologic surveys, such as the ongoing Framingham Study, have not documented any meaningful change in overall death rates. $80 \%$ of men and $70 \%$ of women under age 65 who have CHF will die within 8 years. ${ }^{(1)}$ This is in contrast to the average life expectancy of 19.2 years for women at age 65 and 16.3 years for men (2003 United Nations Economic Commission for Europe report). ${ }^{(2)}$ From 1992-2002 deaths from heart failure increased by $35.3 \%$, while in the same period the overall death rate increased by $7.7 \%{ }^{(2)}$

\section{PATHOPHYSIOLOGY OF HEART FAILURE}

Various conceptual paradigms have been put forward to explain the clinical syndrome of heart failure. The mechanistic view of heart failure as failure to pump blood at a rate required by the metabolizing tissues, or ability to do so only with an elevated pressure, is only partially correct. Research in the past few decades has highlighted the importance of activation of neurohumoral mechanisms, immune system, ventricular remodeling and development of renal changes, i.e. cardiorenal syndrome in the progression of disease. It is beyond the scope of this review to go over the details. Salient features to understand are that I) heart failure is a systemic dynamic progressive disease process brought on by the interaction between the heart, kidneys, renin-angiotensin system, sympathetic nervous system, endothelium and immune system through intricate feedback loops, 2) development of cardiorenal syndrome - the spiral of worsening heart failure and kidney failure that leads to diuretic resistance, volume overload and refractory heart failure, and 3) volume overload plays a significant role in pathophysiology of progressive heart failure apart from contributing to symptomatology. ${ }^{(3,4)}$

\section{MANAGEMENT OF VOLUME OVERLOAD IN HEART FAILURE}

Insights into these pathophysological mechanisms has changed treatment of heart failure in the last two decades, with the introduction of beta blockers and angiotensin converting enzyme (ACE) inhibitors as the first line of treatment and experimental therapies like anti-tumor necrosis factor (TNF) agent, anti-vasopressin and natriuretic peptides.

It is not within the scope of this paper to go into the details of all the therapies for heart failure. We refer the readers to the American College of Cardiology 2005 guidelines..$^{(5)}$ We'll only discuss problems with management of salt and water excretion in refractory heart failure. newer drugs and the place of peritoneal dialysis. The importance of salt and water management is borne out by the fact that $80 \%$ of $\mathrm{CHF}$ admissions are for acute decompensation. Most of these patients are admitted for fluid overload and congestion rather than low perfusion. Only $5 \%$ of patients have low output at admission. (6) Unfortunately, diuretic-based strategies are not always effective in reducing edema. In ADHERE (The Acute Decompensated Heart failure National Registry), $21 \%$ of patients admitted for decompensated heart failure were discharged without weight loss or with a gain in weight.(7)

Salt and water excretion is impaired early in patients with congestive heart failure because of the following reasons:

Low cardiac output leads to underfilling and compensatory increase in sodium and water retention in proximal tubules.

Activation of Renin Angiotensin Aldosterone System(RAAS) causes sodium retention mediated by Angiotensin II (AT 2) through its action on Angiotensin I (ATI) receptor.

Release of aldosterone due to RAAS activation impairs distal tubular sodium excretion. 
- Sympathetic activation leads to sodium and water retention indirectly, by activating the RAAS system and by decreasing the glomerular filtration rate (GFR) secondary to vasoconstriction.

- Impairment of GFR mediated by various neurohormonal pathways; vasoactive molecules impair sodium and water excretion.

Activation of vasopressin impairs water excretion.

Moreover, heart failure patients with mild to moderate disease have a response that is only one-fourth to one-third of that normally observed with maximally effective doses of loop diuretics. The response in patients with more severe disease is smaller yet.(8) This is due to many of the factors described above, which cause salt and water retention apart from impaired absorption of oral drugs due to gastric congestion and increased proximal tubular absorption, with decreased delivery of filtrate to Thick Ascending Limb of Henle (TALH) and distal tubule where the traditional diuretics act.

Apart from failure of therapy, there is concern of increased mortality with use of some classes of diuretics. SOLVD (Studies Of LeftVenricular Dysfunction) database demonstrated that, compared with patients not taking any diuretic, the risk of hospitalization or death due to worsening heart failure in patients taking non-potassium sparing diuretics alone was significantly increased (risk ratio $-1.31,95 \% \mathrm{Cl} 1.09$ to 1.57; $p<0.0004)$ and this was not observed in patients taking potassium sparing diuretics with or without a non-potassium sparing diuretic. ${ }^{(9)}$

Hence the ongoing search for newer agents in the management of salt and water retention. As such, many of the current standard therapies for heart failure directly or indirectly influence sodium and water excretion like beta-blockers, ACE inhibitors, inotropes and aldosterone antagonists. Newer agents like vasopressin receptor antagonists (vaptans), natriuretic peptides and adenosine receptor antagonists being developed for the treatment of heart failure also promote salt and water excretion. Among these, vaptans are the only drugs which are closer to being used in routine clinical practice.

\section{Vasopressin receptor antagonists}

Development of vasopressin receptor antagonists was prompted by the realization that levels of arginine vasopressin are elevated in heart failure and are believed to result in I) myocardial hypertrophy and vasoconstriction mediated by its action on VIa receptor causing vasoconstriction and increasing after load, and 2) water retention and hyponatremia mediated by its action on $\mathrm{V} 2$ receptor and thereby increases preload. Early trials have shown that therapy with vaptans significantly reduces weight, corrects hyponatremia, ${ }^{(10)}$ and reduces mortality. ${ }^{(I)}$ These encouraging findings have led to a large multi-center randomised double blind trial EVEREST (The Efficacy of Vasopressin Antagonism in Heart Failure Outcome Study With Tolvaptan). In this trial, during a median follow-up of 9.9 months, there was no difference in overall mortality, cardiovascular mortality or hospitalization in the group treated with Tolvaptan as compared to placebo in addition to standard therapy. But Tolvaptan significantly improved secondary end points of day I patient-assessed dyspnea, day I body weight, and day 7 edema. In patients with hyponatremia, serum sodium levels significantly increased. ${ }^{(12)}$

\section{Ultrafiltration}

The use of ultrafiltration(UF) for CHF was reported as early as in 1979.(13) However, the need for bulky hemodialysis machines and central venous access made this treatment unpopular. Availability of a new peripheral veno venous hemofiltration machine (system 100, CHF solutions) has brought this treatment into focus again. Costonza et al. ${ }^{(16)}$ reported their experience in 20 patients with acute decompensated heart failure. This machine was used to ultrafilter till the patients were relieved of congestive symptoms. On average, 8.6+ 4.2 L was removed. All patients had relief of all signs and symptoms of CHF and they remained symptom free till 90 days. Treatment was not associated with deterioration in renal parmaeters or hypotension. However, the mean dose of diuretics used increased with admission and remained high after discharge.

In another study Bart et al. (RAPID-CHF trial)(14) reported the results of a randomized but nonblinded trial of usual care versus usual care and UF in 40 patients admitted with acute decompensated heart failure and evidence of volume overload. Patients in the UF group received an 8-h UF treatment in addition to usual care; however, diuretics were withheld during UF. Dyspnea and CHF symptoms improved in both groups at 24 hours, with a slightly greater improvement at 48 hours in the UF group. The average volume removal was higher in the UF group at 24 hours (4.6 vs. $2.8 \mathrm{I}$ ) and 48 hours (8.4 vs. 5.3 I). However, weight loss, a surrogate marker of adequate diuresis and net fluid loss, which was the primary endpoint of the study, was not significantly different between groups. There was one death in the UF group during the 30-day follow-up. Adverse events included catheter infection, which required a 4-week course of intravenous (IV) antibiotics, and there was a small but significant drop in the hemoglobin in the treatment group, the opposite of what could be expected during aggressive diuresis.

The Ultrafiltration Versus IV Diuretics for Patients Hospitalized for Acute Decompensated Congestive Heart Failure (UNLOAD) trial compared the use of intravenous diuretics and ultrafiltration in 200 patients hospitalized for decompensated heart failure. Preliminary data were presented at the 2005 American College of cardiology meeting, and indicated that patients randomly assigned to receive ultrafiltration lost more weight and, at 90 days, had a lower rate of rehospitalization. Incidence of hypotension was similiar in the two groups and ultrafiltration did not seem to have an adverse effect on the renal function. ${ }^{(15)}$

Marenzi g et al. ${ }^{(17)}$ studied the effect of ultrafiltration on hemodynamics in 20 patients with refractory congestive heart failure. All patients underwent ultrafiltration till Hematocrit increased by $10 \%$. The average 
fluid removed was $4.88 \mathrm{I}+896 \mathrm{ml}$. They found that mean right atrial, pulmonary artery and wedge pressures decreased and cardiac output and stroke volume increased during the procedure and all the changes persisted up to 24 hours after procedure.

Recently Gura et al. ${ }^{(18)}$ have reported the development and animal trial of a new wearable ultrafilterable continuous device. The device consists of a hollow fiber filter, a 9 volt battery-operated pulsatile blood pump, a micro pump for heparin infusion, and another micro pump to control ultrafiltration rate. Blood flow used was $65 \mathrm{ml} / \mathrm{min}$ and the weight of the device is less than $2.5 \mathrm{lb}$. Fluid removal rate ranged from 0 to $700 \mathrm{ml} / \mathrm{h}$ and averaged $106 \mathrm{ml} / \mathrm{h}$.

This treatment modality is still in its infancy. Lots of questions still remain unanswered, such as: I) Are there mortality and morbidity benefits? 2) Who are the patients that are likely to benefit and at what cost? 3) Are the benefits sustained? 4) Does it alter the cardiorenal syndrome or worsen it? 5) Does it alter the pathophysiology of heart failure?

\section{PERITONEAL DIALYSIS}

In refractory heart failure the predominant pathophysiology is sodium and fluid retention, and azotemia due to renal hypoperfusion with inadequate response to traditional medical therapy. A logical treatment for this "cardiorenal syndrome" is the use of dialysis, which is efficient in treating both the hypervolemia and azotemia of refractory heart failure. Though all modalities of dialysis have been tried, PD is the simplest choice and offers several advantages. It is an already established longterm home-based therapy and does not require complex machinery or hospital resources. PD is associated with preservation of residual renal function, gentle continuous ultrafiltration, hemodynamic stability, better middle molecule clearance, sodium seiving with maintenance of normonatremia and maybe less inflammation, especially with newer solutions compared to hemodialysis.

Clearance of middle molecules could be of importance. Various cytokines and humoral factors such as TNF, IL-I, reactive oxygen species(ROS), nitric oxide, vasopressin, angiotensin 2 and aldosterone have been implicated in the progression of heart failure. The molecular weight of these substances ranges between 500 and 30000 dalton, which can be removed by PD.Zemel et al.(19) have shown the appearance ofTNF alpha, soluble TNF receptor I and 2 in the PD effluent.

Hyponatremia is a marker for poor outcome in heart failure. Among heart failure patients treated with angiotensin-converting enzyme (ACE) inhibitors, diuretics and beta-blockers, even a small decline in serum sodium levels, to $136 \mathrm{mEq} / \mathrm{L}$ or less, was associated with more than twice the risk of 60-day mortality and a significant increase in risk of readmission or death within 60 days compared with serum sodium levels greater than $136 \mathrm{mEq} / \mathrm{L}{ }^{(20)}$ In a study of patients with end-stage heart failure, Licata $G$ et al., ${ }^{(21)}$ attempted to isolate the effect of an increase in serum sodium on clinical outcome. They randomized 107 patients with refractory heart failure to receive an IV infusion of furosemide plus hypertonic 3\% saline solution or an IV bolus of furosemide twice a day without hypertonic saline. Survival over a mean follow-up of 31 months was $55 \%$ in the group that received hypertonic saline compared with 13\% in those that did not receive hypertonic saline $(P<.00 l)$. This suggests that normalization of a low serum sodium by sodium seiving may be another potential mechanism of benefit for PD.

Renal insufficiency significantly increases the risk of death and thus is an important prognostic indicator in heart failure patients. Hillege et al. ${ }^{(22)}$ reported in the Second Prospective Randomized Study of Ibopamine on Mortality and Efficacy (PRIME-2) that patients with GFRs in the lowest quartile $(<44 \mathrm{~mL} /$ minute) had almost a three times higher risk of mortality than those in the highest quartile (GFR $>76$; relative risk $2.85, \mathrm{P}<.000 \mathrm{I}$ ). Impaired renal function was a stronger predictor of death in these patients with heart failure than a low ejection fraction. Forman et al. ${ }^{(23)}$ found that heart failure patients whose renal function worsened while in the hospital had longer stays, incurred higher hospital costs, were more likely to die in the hospital and, if they survived the hospitalization, were more likely to be readmitted. It is not very clear whether this is an association because patients with renal failure are more likely to have refractory heart failure or if preserved renal function protects from worsening heart failure due to less inflammatory activation. PD again has an advantage by preserving residual renal function.

In 1949, Schneierson ${ }^{(24)}$ published the first case report using PD as a successful therapy in a patient with severe CHF. Since then there have been multiple case reports and small series. Review of use of PD in CHF can be divided into three periods - intermittent PD(IPD), continuous $\mathrm{PD}$ and newer soultions. During these three phases, not only the technology of PD has changed, but also treatment of CHF has changed.

\section{Intermittent PD}

In the 1960s many cases of acute CHF treated by PD have been reported, in total about 56 such patients. In summary these reports demonstrated fluid removal rate of $67-568 \mathrm{ml} /$ hour with improvement in symptoms in most of the patients. Fluid removal was associated with an improvement in plasma volume ${ }^{(25)}$ and hyponatremia. ${ }^{(26)}$ But the effect on cardiac output was variable. Interestingly a significant proportion of patients became diuretic responsive and their renal function improved. Shilo et al. ${ }^{(27)}$ demonstrated significant improvement in GFR as measured by creatinine and inulin clearance and also renal blood flow measured by $\mathrm{PAH}$ clearance in 9 patients with refractory CHF who underwent intermittent PD. But because of the acute and intermittent nature of the treatment provided, it did not change the long-term course of the disease. Patients with remediable disease like acute myocardial infarction or the ones who went for cardiac surgery benefited from the ultrafiltration. Raja et al. ${ }^{(28)}$ first reported the use of repeated intermittent PD in a 59-year-old patient with refractory CHF. 
The patient underwent a total of 8 treatments over a period of 2 years and eventually died in a car accident. But later reports were not so optimistic. Shapira et al. ${ }^{(29)}$ reported repeated intermittent PD in 10 patients with refractory CHF.Though all patients showed improvement in symptomatology, sodium levels, urine output and diuretic responsiveness, $50 \%$ of the patients died 99 to 354 days after their first dialysis. $90 \%$ of their patients also had gram negative bacteria cultured from their PD fluid but none of the patients developed peritonitis. Another major limitation of this therapy was requirement of frequent hospitalization for the procedure.

\section{Continuous PD}

Despite the initial disappointment with IPD in the long-term management of heart failure, it was clear that Continuous PD (CAPD), because of its continuous nature, will be more appropriate. There are multiple case series on the use of CAPD and we summarise the major trials in the Table I. (NO TABLE SUPPLIED)

As we can see from the table, all patients show symptomatic improvement while on PD. Hospitalisation rate was decreased in all studies except the one reported by Rubin et al. ${ }^{(33)}$ In their study patients were hospitalized as often for PD-related problems as for cardiac problems. Peritonitis rates were high in the earlier studies, which is not the problem with the newer connecting devices and automated devices.

Though all patients showed symptomatic improvement, mortality remained high. It is not clear whether better volume management will translate to delay in progression of heart failure. There was no correlation between functional improvement and LV function as measured by left ventricular ejection fraction (LVEF). ${ }^{(31)}$ But Stegmayr et al. ${ }^{(35)}$ have shown reduction in CT index and Gotloib et al. ${ }^{(39)}$ in their study found an improvement in the cardiac work index. Long-term follow-up studies are required to see the effect on progression.

Though mortality was high in most of the studies, survival seems to be improving in the recent trials. Stegmayr et al. ${ }^{(35)}$ studied patients with expected survival of I month and showed a significant prolonged survival from I month up to I year. In the study by Gotloib et al. ${ }^{(39)}$ they found a I-year mortality rate of I0\%, which was significantly less than the $80 \%$ expected based on their charles comorbidity index. It is difficult to interpret these mortality data because none of these trials compared PD to standard care of therapy. It will be not be possible to do such a trial in patients with refractory heart failure and, for that matter, cardiac transplantation has never been compared either. It is also difficult to interpret whether this improvement is because of PD or overall improved management and survival in CHF patients with newer therapies.

It is also interesting to note that in the trial by Ryckelynck et al. ${ }^{(36)}$ two patients who were earlier rejected for cardiac transplant were found to be fit and got transplanted after starting PD. In the trial by Konig et al.(.34) three patients had cardiac transplantation after starting PD. PD could be afforded to patients waiting for cardiac transplant as bridge therapy.

\section{Newer PD solutions}

Even in renal failure patients on PD, introduction of icodextrin has been shown to decrease extra cellular water and improve hemodynamics. Icodextrin offers several advantages:

It offers more physiologic ultrafiltration.

Euvolemia can be maintained without additional dextrose exchanges.

Single exchange is easy on lifestyle and decreases risk of touch contamination and hence incidence of peritonitis.

Avoidance of dextrose solutions could mean less peritoneal inflammation. The effect of such inflammatory markers in the progression of heart disease is not known.

Konings et al. ${ }^{(41)}$ randomised 40 CAPD/CCPD patients (renal failure patients on dialysis) to either icodextrin during long dwell or standard glucose solution and found that use of icodextrin was associated with increased ultrafiltration, lower extracelluar water as estimated by bromide dilution method. At the end of 4-month follow-up period they were also found to have significantly lower LV mass. There are only a few case reports on the use of icodextrin in treatment of refractory $\mathrm{CHF}$. Bertoli et al. ${ }^{(42)}$ reported the use of a single 12-hour nighttime manual CAPD exchange with icodextrin in 2 non-uremic elderly patients with NYHA class 3-4 failure. They had daily ultrafiltration of 500- I 000 ml. Both patients showed improvement in their NYHA class, ejection fraction after $12-15$ months on PD. Urine output declined modestly in one patient, but renal function measured as creatinine clearance by the Cockroft-Gault method improved in both the patients. Neither of the patients required hospitalization for either cardiac or dialysis related issues, as compared to multiple admissions in the preceding year.

\section{FUTURE PROSPECTS}

- Comparison of PD versus standard therapy and ultrafiltration devices to demonstrate survival benefit with less morbidity and costs.

- Is there preservation of renal function with PD as compared to standard medical therapy? Will this delay the progression of heart failure by interrupting cardiorenal syndrome?

What will be the effect of biocompatible PD solution with associated less inflammation on heart failure pathogenesis?

Large trials with long-term follow-up looking at the effect of PD on progression of heart failure. 


\section{REFERENCES:}

1. American heart association. Heart disease and stroke statistics: 2005 update www.americanheart.org

2. Trends in Europe and North America. The statistical yearbook of the economic commission for europe 2003. www.unece.org/stats/trend/trend_h.html, 2003.

3. Jessup M, Brozena S. Heart failure. N Engl J Med 2003; 348: 2007-20I 8

4. Francis G. Acute decompensated heart failure: the cardiorenal syndrome. Cleve Clin J Med 2006; 73 Suppl 2: S8-13.

5. Hunt SA: Acc/aha 2005 guideline update for the diagnosis and management of chronic heart failure in the adult: a report of the American college of cardiology/American heart association task force on practice guidelines (writing committee to update the 200 guidelines for the evaluation and management of heart failure). J Am Coll Cardio 2005; 46: el-82.

6. Gheorghiade M, Zannad F, Sopko G et al. Acute heart failure syndromes: current state and framework for future research. Circulation 2005; I I 2: 3958-3968.

7. Adhere $^{\mathrm{TM}}$ (acute decompensated heart failure national registry). Third quarter 2004 national benchmark report. www.adhereregistry.com/nat.html.

8. Brater DC, Day B, Burdette A, Anderson S. Bumetanide and furosemide in heart failure. Kidney international 1984; 26: 183-189.

9. Domanski M, Norman J, Pitt B, Haigney M, Hanlon S, Peyster E. Diuretic use, progressive heart failure, and death in patients in the studies of left ventricular dysfunction (SOLVD) J Am Coll Cardiol 2003; 42: 705-708.

10. Gheorghiade M, Niazi I, Ouyang J et al.Vasopressin v2-receptor blockade with tolvaptan in patients with chronic heart failure: results from a double-blind, randomized trial. Circulation 2003; 107: 2690-2696

II. Gheorghiade M, Gattis WA, O'Connor CM et al. Effects of tolvaptan, a vasopressin antagonist, in patients hospitalized with worsening heart failure: a randomized controlled trial. JAMA 2004; 291: 1963-1971.

12. Konstam MA, Gheorghiade M, Burnett JC Jr et al. Effects of Oral Tolvaptan in Patients Hospitalized for Worsening Heart Failure.The EVEREST Outcome Trial. JAMA 2007; 297:|3|9-|33|

13. Gerhardt RE, Abdullah AM, Mach SJ, Hudson JB. Isolated ultrafiltration in the treatment of refractory congestive heart failure. 1979; 139: 358-359

14. Bart BA, Boyle A, Bank AJ et al. Ultrafiltration versus usual care for hospitalized patients with heart failure: the relief for acutely fluid-overloaded patients with decompensated congestive heart failure (RAPID-CHF) trial. J Am Coll Cardiol 2005; 46: 2043-2046

15. Cleland JGF, Coletta AP, Nikitin NP, Clark AL. Clinical trials update from the American College of Cardiology: darbepoetin alfa, asteroid, universe, paediatric carvedilol, unload and iceland. Eur I Heart Fail 2006; 8: 326-329.

16. Costanzo MR, Saltzberg M, O'Sullivan J, Sobotka P. Early ultrafiltration in patients with decompensated heart failure and diuretic resistance. J Am Coll Cardiol 2005; 46 : 2047-2051.

17. Marenzi G, Lauri G, Grazi M, Assanelli E, Campodonico J, Agostoni P. Circulatory response to fluid overload removal by extracorporeal ultrafiltration in refractory congestive heart failure. J Am Coll Cardiol 200 I; 38: 963-968.

18. Gura V, Beizai M, Ezon C, Rambod E. Continuous renal replacement therapy for congestive heart failure: the wearable continuous ultrafiltration system. ASAIO J 2006 52:59-61.

19. Zemel D, Imholz AL, De Waart DR, Dinkla C, Struijk DG, Krediet RT. Appearance of tumor necrosis factor-alpha and soluble tnf-receptors $\mathrm{i}$ and ii in peritoneal effluent of CAPD. Kidney Int 1994; 46: I422-1430.

20. Klein L, O'Connor CM, Leimberger JD et al. Lower serum sodium is associated with increased short-term mortality in hospitalized patients with worsening heart failure: results from the outcomes of a prospective trial of intravenous milrinone for exacerbations of chronic heart failure (OPTIME-CHF) study. Circulation 2005: | I : 2454-2460

21. Licata G, Di Pasquale P, Parrinello G et al. Effects of high-dose furosemide and smallvolume hypertonic saline solution infusion in comparison with a high dose of furosemide as bolus in refractory congestive heart failure: long-term effects. Am Heart | 2003; 145: 459-466.

22. Hillege HL, Girbes AR, De Kam PJ et al. Renal function, neurohormonal activation, and survival in patients with chronic heart failure. Circulation 2000; 102: 203-210.

23. Forman DE, Butler J, Wang $Y$ et al. Incidence, predictors at admission, and impact of worsening renal function among patients hospitalized with heart failure. J Am Coll Cardiol 2004: 43: 61-67.
24. Schneierson SI. Continuous peritoneal irrigation in the treatment of intractable edema of cardiac origin. The American Journal of the Medical Sciences 1949; 21 8: 76-79.

25. Cairns KB, Porter GA, Kloster FE, Bristow JD, Griswold HE. Clinical and hemodynamic results of peritoneal dialysis for severe cardiac failure. American Heart Journal 1968 76: 227-234

26. Malach M. Peritoneal dialysis for intractable heart failure in acute myocardial infarction The American Journal of Cardiology 1972; 29:61-63.

27. Shilo S, Slotki IN, laina A. Improved renal function following acute peritoneal dialysis in patients with intractable congestive heart failure. Israel Journal of Medical Sciences 1987; 23: $821-824$.

28. Raja RM, Krasnoff SO, Moros JG, Kramer MS, Rosenbaum JL. Repeated peritoneal dialysis in treatment of heart failure. IAMA :The journal of the American Medical Association 1970; 21 3: 2268-2269.

29. Shapira J,Lang R, Jutrin I, Robson M, Ravid M. Peritoneal dialysis in refractory congestive heart failure. Part I: Intermittent peritoneal dialysis. Peritoneal Dialysis Bulletin 1983 Jul-Sep: | 30- 132

30. Robson M, Biro A, Knobel B, Shai G, Ravid M. Peritoneal dialysis in refractory congestive heart failure. part II: Continuous ambulatory peritoneal dialysis (CAPD). Peritoneal Dialysis Bulletin 1983; Jul- Sep: I33- 134.

31. Kim D, Khanna R,Wu G et al. Successful use of continuous ambulatroy peritoneal dialysis in refractory heart failure. Peritoneal Dialysis International 1985; 5: I 27- 130.

32. McKinnie J], Bourgeois RJ, Husserl FE. Long-term therapy for heart failure with continuous ambulatory peritoneal dialysis. Arch Intern Med 1985; I 45: I I 28 - I 129.

33. Rubin J, Ball R. Continuous ambulatory peritoneal dialysis as treatment of severe congestive heart failure in the face of chronic renal failure. Report of eight cases. Arch Intern Med 1986; | 46: I533-1535.

34. Konig PS, Lhotta K, Kronenberg F, Joannidis M, Herold M. CAPD: A successful treatment in patients suffering from therapy-resistant congestive heart failure. In: Khanna R, Nolph KD, Prowant BF, Twardowski ZJ, Oreopoulos DG, eds. Advances in Peritoneal Dialysis. Toronto: Peritoneal Dialysis Bulletin 1991: 7:97-101.

35. Stegmayr BG, Banga R, Lundberg L, Wikdahl AM, Plum-Wirell M. PD treatment for severe congestive heart failure. Peritoneal dialysis international: journal of the International Society for Peritoneal Dialysis 1996; I 6 Suppl I: S23I-235.

36. Ryckelynck JP, Lobbedez T, Valette B et al. Peritoneal ultrafiltration and refractory congestive heart failure. Advances in Peritoneal Dialysis. Conference on Peritoneal Dialysis 1997: 13: 93-97.

37. Tormey V, Conlon PJ, Farrell J, Horgan J,Walshe J]. Long-term successful management of refractory congestive cardiac failure by intermittent ambulatory peritoneal ultrafiltration. QJM: monthly journal of the Association of Physicians 1996; 89: 68I-683.

38. Ortiz AM, Acosta PA, Corbalan R, Jalil JE. Long-term automated peritoneal dialysis in patients with refractory congestive heart failure. Advances in Peritoneal Dialysis. Conference on Peritoneal Dialysis 2003; 19: 77-80.

39. Gotloib L, Fudin R, Yakubovich M, Vienken J. Peritoneal dialysis in refractory end-stage congestive heart failure: A challenge facing a no-win situation. Nephrol Dial Transplant 2005; 20 Suppl 7: vii32-vii36.

40. Kagan A, Rapoport J.The role of peritoneal dialysis in the treatment of refractory hear failure. Nephrol Dial Transplant 2005; 20 Suppl 7: vii28-vii3।

4I. Konings CJAM, Kooman JP, Schonck M et al. Effect of icodextrin on volume status, blood pressure and echocardiographic parameters: a randomized study. Kidney Int 2003; 63: 1556-1563.

42. Bertoli SV. Ciurlino D, Maccario M et al. Home peritoneal ultrafiltration in patients with severe congestive heart failure without end-stage renal disease. Advances in Peritoneal Dialysis. Conference on Peritoneal Dialysis 2005: 21: 123-127. 